\title{
Adaptive Methods of Computing Mathematics and Mechanics Stochastic Variant
}




\title{
Adaptive Methods of Computing Mathematics and Mechanics Stochastic Variant
}

\author{
D. G. Arsenjev, \\ V. M. Ivanov \& O. Y. Kul'chitsky
}

St. Petersburg State Technical University, Russia 


\section{Published by}

World Scientific Publishing Co. Pte. Lid.

P O Box 128, Farrer Road, Singapore 912805

USA office: Suite 1B, 1060 Main Street, River Edge, NJ 07661

UK office: 57 Shelton Street, Covent Garden, London WC2H 9HE

\section{Lbrary of Congress Cataloging-in-Publication Date \\ Arsen'ev, D. G. (Dmitrii Germanovich), 1963-}

[Adaptivnye metody vychislitel 'noi matematiki i mekhaniki.

English]

Adaptive methods of computing mathematics and mechanics :

stochastic variant / by Dmitry G. Arsenjev, Vladimir M. Ivanov, Oleg

Y. Kul'chitsky; [A.P. Sokolov, translator].

p. $\mathbf{c m}$.

Includes bibliographical references and index.

ISBN 9810235011

1. Mechanics, Analytic -- Data processing. 2. Multigrid methods

(Numerical analysis). 3. Mathematical models. I. Ivanov, V. M.

(Vladimir Mikhailovich), 1956- . II. Kul'chitskiì, O. IU. (Oleg

IU'evich), 1946- . III. Title.

QA808.A7713 1998

$531-\mathrm{dc} 21$

98-7861

CIP

\section{British Library Cataloguing-in-Publication Data}

A catalogue record for this book is available from the British Library.

Copyright 1999 by World Scientific Publishing Co. Pte. Ltd.

All rights reserved. This book, or parts thereof, may not be reproduced in any form or by any means. electronic or mechanical, including photocopying, recording or any information storage and retrieval system now known or to be invented, without written permission from the Publisher.

For photocopying of material in this volume, please pay a copying fee through the Copyright Clearance Center, Inc., 222 Rosewood Drive, Danvers, MA 01923, USA. In this case permission to photocopy is not required from the publisher.

This book is printed on acid-free paper.

Printed in Singapore by Uto-Print 


\section{Foreword}

Modern scientific research in various fields are held by means of mathematical modeling, which uses methods of computing mathematics and employs computers. These research in mechanics have caused an extensive development of a new trend in science, called computation mechanics.

Computation mechanics is tended to come to series of numerical experiments while increasing complexity of mechanical models. The typical case is an experiment with a model of continuous media, analysis of which demands grid methods to be applied. It may be conducted, for example, when designing constructions for extreme operational conditions. This experiment turns out to be complicated and labor-consuming, requiring large expenditures in terms of both intellectual and computing resources. In a number of cases, when spatial constructions of complex configuration are being calculated, complex computation problems occur, in particular, the so-called «curse of dimensionality (deriving numerical solutions of these problems in mechanics requires solution of equation sets of huge dimensionality of $\left(10^{3}-10^{6}\right)$, which becomes a source of evaluation errors).

Advanced computing facilities have been expanded the scope of admissible complexity of problems to be solved. However, the researchers' demands and the complexity of problems to be solved grow so rapidly that available hardware usually cannot satisfy them.

In this connection one can justly wonder:

\section{Does this problem have algorithmic reserves?}

If it has, what are they?

The answer to the first question is apparently positive. The most worthwhile field of the grid methods for such reserves to be looked for is a special non-uniform organization of integration grid. In general, it seems quite obvious, that it is sufficient to construct a grid, which is denser in those parts of integration domain, where the intensity of solution varying is the greatest, to reach the highest accuracy of solution on a grid with the same number of nodes. Heuristic algorithms of integration grid optimization, designed in the sense of these principles, guarantee significant economy of computing resources, so they are widely spread in computation mechanics. 
Thus, basis of the methods of rational selection of the grid nodes coordinates is formed by an a priori information concerning the properties of solutions being sought. It is also possible to set precisely the problem of an optimal choice of the grid nodes coordinates as a problem of mathematical programming with optimizing function and restrictions depending on functions being sought. However, there are at least two serious difficulties in solving such a problem. These difficulties were overcome only recently.

First, the problem of optimal choice of integration grid as a problem of mathematical programming cannot be solved a priori, its very setting demands knowing the fields of tensions or deformations to be sought.

Second, dimensionality and complexity of solution of this problem, even with known fields of tensions and deformations being sought, several times exceed dimensionality of solution of initial problem of evaluation of fields sought for: since the result of grid optimization is a set of optimal grid node coordinates, quantity of variables to be optimized two or three times exceed quantity of unknown values of initial problem, we do not even mention the complicated structure of restrictions these coordinates should meet.

Thus, the problem of integration grid optimization encounters problems which cannot be solved by means of traditional methods of computing mathematics. It is evident that new fundamental ideas are needed for this field to expand.

In the first part of this monograph two such ideas are put forward and developed: an idea of adaptation and an idea of statistical computing mathematics.

The idea of adaptation is grounded with the ideas and methods of adaptive control, which are well developed in the theory of control. With regard to the problem of grid optimization it means in the simplified form, that the process of grid selection in integration algorithm should be iterative and should alternate with the process of solution evaluation. Here recurrent specified evaluation of fields being sought is to be an a priori information for solution of optimization problem of correcting grid characteristics. The ideas of adaptation have already been used in computing methods implicitly.

The second idea is less traditional for computing mathematics and traces its roots to the fields of statistical mechanics and physics, thermodynamics and the theory of vibration conductivity. So, when investigating mechanical systems with large number of degrees of freedom, like gases, liquids, other dynamic systems it is senseless to consider behavior of a system for each particular degree of freedom; mathematical models of such systems should be generalized and integrated instead, their behavior should be described in ensemble characteristics, like density function, adjoined distribution function, 
expectation, variance, etc.

To attain high accuracy when solving the problems of continuous media mechanics or heat conduction numerically it is custom to use grid methods with a large number of grid nodes, which exceeds tens of thousands in some problems. We propose not to regard the grid nodes as particular objects, but to consider their density function in domain of integration as an ensemble characteristics, similarly to the way problems are used to be set in statistical mechanics. To put it in another way, the particular location of nodes does not matter; it is their relative density of concentration within the field of integration that is significant.

The idea of ensemble description of characteristics of integration grid nodes allows one to transform a mathematical programming problem, concerning selection of the optimal grid, into the corresponding variational problem. In a number of cases this problem can be solved in analytical form, unlike the problem of mathematical programming. In other cases it can only be solved approximately. However, the solution complexity is considerably less than that of a similar problem of mathematical programming, since in a variational optimization problem ordering-type restrictions on node coordinates, which are complex for numerical implementation, turn out to be taken into account automatically, and high accuracy of definition of nodes distribution density is out of need for approximations to be "good". There are the trends of their concentration that is of importance. That is why trivial step approximation of distribution density is sufficient.

It is necessary to note, that the idea of statistical description of integration grid characteristics does not solve the problem of dependence of variational problem on the solution being sought. For this reason both ideas can work in the capacity of statistical description of nodes only when they are applied together. The set of these ideas, methods and algorithms is what authors call statistical computing mathematics.

Numerical methods of Monte-Carlo, also used to be called methods of statistical trials, are well-developed kin of statistical computing mathematics. The theory of these methods is analyzed in details in fundamental monographs by I.M. Sobol, S.M. Ermakov, G.A. Mikhailov, V.B. Melas, A.A. Zhigliavsky, V.V. Nekrutkin, B.S. Elepov, A.S. Sipin, K.K. Sabelfeld and others. In the present monograph these methods, supplemented by adaptation methods and applied to solution of integral equations and boundary-value problems of mechanics, are considered as a basis for the new approach in designing the algorithms of statistical computing mathematics.

Methods of statistical trials have a number of unique algorithmic abilities. In particular, these methods and their errors of evaluation depend on 
dimensionality of integration domain only weakly, the process of forming integration grid can be easily automatized, and, coupled with computing algorithm, can be performed in recurrent form or in form of parallel computation. Another ability is that evaluation structure provides a basis for designing special software for multiprocessor computers.

Until recently, use of statistical methods in computation mechanics was limited by two circumstances: convergence, slow in comparison with deterministic methods, and insufficient development or lack of algorithms for solution of problems of elasticity and heat conduction which often occur in engineering practice.

The present monograph fills in these gaps. It presents statistical algorithms for the problems, which have not been solved by the similar methods earlier; the algorithms, known from publications, were improved, which provides greater speed of their convergence and an opportunity of complete automatization of computing processes while solving the initial problems of computing mathematics. It was reached due to using the effective determinate procedures in statistic algorithms, and to creation and application of special adaptive computing methods.

The so-called semi-statistical method is one of them. It combines both statistical and determinate operations and is intended for numerical solution of integral equations. The method enables recurrent increase of number of nodes in a random integration grid, optimization of its structure and control over accuracy of estimates obtained while performing process of evaluation.

When developed semi-statistical method, an auxiliary, but significant problem has being solved; namely, the problem of adaptive integral evaluation. The obtained class of adaptive statistical algorithms of numerical integration has convergence speed similar to the deterministic analogies, while traditional statistical methods provide much slower convergence.

Another group of methods discussed here bears the name of the random walk methods (RWM). These methods enable one to determine tension or temperature on just one or several points of the body instead of defining the whole field of solutions. RWM is convenient for use in problems, which require only determination of experimental values of solution, while their domain is known. An overwhelming majority of the machine-building problems on finding maximal tension or temperature fits this condition, since the location of dangerous zones within a construction is usually well-known.

The last part of the monograph concerns application of main ideas of the book to finite elements method (FEM). It is devoted to rational selection of FEM random grid. One-dimensional engineering problems have been selected as examples. Nodes of FEM grid are random. Distribution density is defined 
by minimum condition of potential energy of one-dimensional elastic system, expressed by desired nodal values of transfer.

This monograph consists of three parts. The first part concerns development of adaptive-statistical methods of integral evaluation and solution of integral equations, as well as application of these methods to the problems of continuous media mechanics; the second part concerns development of RWMs for solution of boundary-value problems of the elasticity theory and the heat conduction theory. The third part concerns application of main ideas of the book to finite elements method.

In Chap. 1 the main ideas and approaches of the Monte-Carlo method, known in literature, are discussed. This chapter is introductory and auxiliary for further discussion.

In Chap. 2 the theory of adaptive-statistical integral evaluation is discussed. It is shown that this class of methods of integral evaluation has an increased convergence speed, which is close to the conversion speed of respective deterministic analogies, although it possesses such advantages of statistical methods as recurrence in both evaluation of the integral itself and level of achieved accuracy. Theoretical research of the properties of these algorithms was refined by numerical experiments, which have shown even greater efficiency of the methods, than one proposed by the theory.

In Chap. 3 the theory of semi-statistical method of numerical solution of integral equations is discussed. Algorithmic abilities of the method are considered, formulas of recurrent representation are derived. Convergence of the proposed algorithms is proved, the matter of grid optimization is investigated.

Chapter 4 discusses a variant of the semi-statistical method, so-called projection-statistical method.

In Chap. 5 application of the semi-statistical method to the problem of vibration conductivity is discussed. Integral equations were derived for the problem under consideration. Abilities of the method and efficiency of adaptive optimization of a random grid structure are demonstrated using a number of examples.

In Chap. 6 peculiarities of application of semi-statistical method to solution of integral equations of the first basic problem of elasticity theory are discussed. Corresponding equations are represented in invariant form. Analytical solutions of the integral equation are defined for some classical areas (full sphere, spherical concavity, hollow sphere). Results of numerical modeling on test problems are presented.

In Chap. 7 application of the semi-statistical method to solution of integral equations of the second basic problem of the elasticity theory is discussed. Weyl's integral equations used here are represented in invariant form. 
New method of solution of integral equations for the non-stationary problems is proposed in Chap. 8. Special attention is paid to the most critical part of the general algorithm, namely to integration of the rigid systems of linear differential equations.

Part II (Chaps. 9 - 14) concerns development of the random walk method (RWM) for evaluation of solution of boundary-value problems of the elasticity and heat conduction theory in some points, like the extreme points.

Chapter 9 contains a review of available results, and the main ideas and approaches of the RWM, which are developed in further chapters.

In Chap. 10 application of the RWM to solution of the heat conductivity problems, which can be reduced to Dirichlet or Neumann's boundary problems, is discussed. Along with traditional RWM algorithm to be applied to circles, new RWM algorithms to be applied to circles with displaced center, and RWM with partial integration over a circle are described, and conditions of convergence of these algorithms and errors of their evaluation are obtained. New approaches are compared with traditional ones on results of numerical simulation.

In Chap. 11 application of RWM for solution of the problems of curving of supported and fastened plates with a linear-piecewise and arbitrary contour is discussed. The necessary integral representation is derived for solutions of corresponding boundary-value problems of plate curving. The reasons of divergence of traditional statistical procedures are determined. New algorithms are offered and their convergence is substantiated. Efficiency of the procedures is illustrated by means of numerical simulation.

In Chap. 12 RWM is applied to solution of the flat problems of elasticity theory. Description of the algorithm and results of its application to the test problems are provided.

Chapter 13 illustrates application of RWM to engineering problems of elasticity theory. Problems of finding tension in dangerous points of rigid- or flexible-rimmed cog-wheels, as well as the problem of selecting the best gear tool, are solved. Tensions obtained by means of RWM are compared with results obtained by finite elements method.

In Chap. 14 RWM is used to solve the spatial problem of heat conductivity. Since a special case is considered, namely the heating of a stamp, which geometry is a set of parallelepipeds, it was considered appropriate to develop a method of walking over parallelepipeds, instead of spheres, to increase convergence. The necessary integral representations for a parallelepiped are obtained. An appropriate RWM algorithm is developed, analysis of its convergence is performed, and the influence of statistical and systematic errors on evaluation is examined. 
Part III (Chaps. 15-21) is devoted to rational selection of random grid for solution of a problem by means of finite elements method.

Chap. 15 is the introductory.

In Chap. 16 analytical representations for functionals of expectations of approximations of functionals of potential energy are derived for a rod contour approximated in various ways.

In Chap. 17 analytical expressions for optimal distribution density is derived under large number of nodes.

Chapter 18 is to derive step representation of functionals, defined in Chap. 16 , with a view to minimize them numerically with respect to parameters of step density. Properties of solutions, constructed on optimal grid, are analyzed.

Chapters $19-21$ are devoted to Bemm optimal nodes problem and include theoretical and numerical results.

And finally, a brief survey of the results obtained in the monograph is laid out, and prospective directions of their further development are mentioned.

In conclusion the authors would like to make the following note. This monograph is a result of work carried out by a team of researchers for more than 20 years. The first edition of the book have been published in 1996 by "Nauka", Russia. In this edition, Chap. 4 and Part III have been added. Errors and misprints have also been corrected.

The authors would like to thank Prof. V.A. Palmov for constant support of their research and fruitful discussion on the results obtained. The authors also thank Prof. A.A. Pervozvansky, Prof. L.B. Clebanov, Prof. V.B. Melas and Prof. P.I. Perlin for friendly criticism, which has positively affected the results of the work. And also to Prof. Lennart Ljung for the help in publishing the book.

A number of sections of the manuscript were improved due to careful survey made by post-graduate students D.V. Butenina and M.L. Korenevsky. Furthermore, D.V. Butenina is co-author of Sec. 2.4 and Part III and M.L. Korenevsky is co-author of Chap. 4.

The authors also thank A.P. Sokolov, who translated the book.

Information of great value was received from D.G. Kirian.

And, finally, the monograph would not be published without the help of I.I. Ivanova and S.A. Ivanov. 


\section{Contents}

Foreword

Part I. Evaluation of integrals and solution of integral equations

Chapter 1. Fundamentals of the Monte-Carlo method 3

1.1. Idea of the Monte-Carlo method . . . . . . . . 3

1.2. Simulation of implementation of a scalar random variable . . . . . . . . . . 6

1.2.1. The transforming functions method . . . . . 6

1.2.2. The superposition method $\ldots \ldots \ldots \ldots .9$

1.2.3. The selection method ........... 11

1.3. Simulation of implementation of a vector random variable . . . . . . . . . . . . . . 14

1.4. Evaluation of definite integrals

by means of Monte-Carlo method . . . . . . . 17

Chapter 2. Evaluation of integrals by means of statistic simulation employing adaptation

2.1. Adaptation idea in statistic methods of numerical analysis, based on the principles of importance sampling . . . . . . . . . . . 21

2.2. Adaptive algorithm for evaluating one-dimensional integral . . . . . . . . . . . 23

2.2.1. Selection of probability densities . . . . . . 23

2.2.2. Evaluation procedure . . . . . . . . . 27

2.2.3. Results of numerical experiments . . . . . . 28

2.2.4. Report on the results . . . . . . . . . . 29

2.3. Adaptive algorithm of evaluation of two-dimensional and multi-dimensional integrals . . . . . . . . . 32

2.3.1. Description of the algorithm . . . . . . . 32 
2.3.2. Results of numerical experiments . . . . . . 36

2.3.3. Some comments ............. 36

2.4. Stochastic computing algorithms as anject

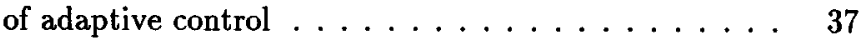

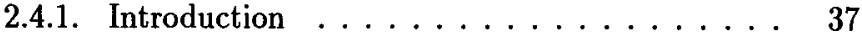

2.4.2. Statement of a problem of control over the process of computation .......... 38

2.4.3. Synthesis of the optimal control over the process of computation ............ 42

2.4.4. Strategy of adaptive optimization of computation process ........... 47

Chapter 3. Semi-statistical method of numerical solving integral equations

3.1. Introduction . . . . . . . . . . . . . . . 49

3.2. Basic relations of the method . . . . . . . . . 50

3.3. Recurrent inversion formulae ........... 52

3.4. Convergence of the method ........... 55

3.5. Adaptive abilities of the algorithm . . . . . . . 70

3.6. Qualitative considerations concerning connections between the semi-statistical and variational methods . 72

3.7. Application of the method to singular integral equations ............... 72

3.7.1. Description and application of the method ............... 72

3.7.2. Recurrent inversion formulae ........ 76

3.7.3. Analysis of the method's errors . . . . . . . 77

3.7.4. Adaptive abilities of the algorithm ...... 80

Chapter 4. Projection-statistical method of numerical solution of integral equations

4.1. Introduction . . . . . . . . . . . . . . 82

4.2. Basic relations of the method . . . . . . . . . . 82

4.3. Formulae of recurrent inversion . . . . . . . . . . 86

4.4. The algorithm convergence ........... 88

4.5. Merits of the method . . . . . . . . . . . . . 99

4.6. Adaptive abilities ... . . . . . . . . . . . 99

4.7. Peculiarities of numerical implementation . . . . . . 101

4.8. An alternative computing technique:

approximate solutions should be averaged ...... 103

4.9. Numerical experiments . . . . . . . . . . . . 105 
4.9.1. A test problem . . . . . . . . . . 105

4.9.2. The problem on steady-state forced small transverse vibration of pinned string caused by harmonic force . . . . . . . . 110

Chapter 5. The problem of vibration conductivity 116

5.1. The boundary-value problem of vibration conductivity 116

5.2. Integral equations of vibration conductivity . . . . 118

5.3. Regularization of the equations . . . . . . . . . 124

5.4. Integral equations with improved asymptotic properties at small $\beta \ldots \ldots \ldots \ldots$

5.5. Numerical solution of the vibration conductivity problems . . . . . . . . . 134

5.5.1. Solution of a test problem . . . . . . . . . 134

5.5.2. Research of the influence of distortion of a sphere and character of an external load on the results of numerical solution . . . . . . . 138

Chapter 6. The first basic problem of the elasticity theory

6.1. Potentials and integral equations of the first basic problem of the elasticity theory . . . 142

6.1.1. The force and pseudo-force tensors . . . . . 142

6.1.2. Integral equations of the first basic problem . . 145

6.2. Solution of some space problems of elasticity theory using the method of potentials . . . . . . . . 146

6.2.1. Solution of the first basic problem for some centrally symmetrical spatial areas . . . . 146

6.2.2. Solution of the first basic problem for a ball . . 148

6.2.3. Solution of the first basic problem for an unlimited medium with a spherical cavity . 149

6.2.4. Solution of the first basic problem for a hollow ball . . . . . . . . . . . 149

6.3. Using semi-statistical method for solution of integral equations of the elasticity theory . . . . 151

6.4. Formulae for optimal density . . . . . . . 155

6.5. Results of numerical simulation . . . . . . . . . 157

Chapter 7. The second basic problem of the elasticity theory 161

7.1. Fundamental solutions of the first and

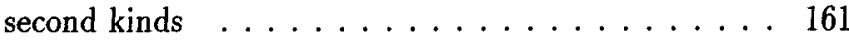

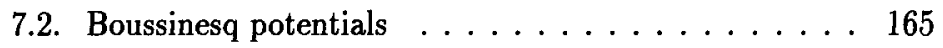


7.3. Weyl tensor . . . . . . . . . . . 166

7.4. Weyl force tensors . . . . . . . . . . 168

7.5. Arbitrary Liapunov surface . . . . . . . . . 170

Chapter 8. A way to solve non-stationary problems 171

8.1. The general scheme of solution of non-stationary integral equation . . . . . . . 171

8.2. A way to integrate systems of linear differential equations . . . . . . . . . . . 175

8.2.1. Setting the problem ........... 175

8.2.2. Estimating an error of evaluation of matrix exponential. . . . . 177

8.2.3. Optimization of the algorithm of approximate computation of matrix exponential 181

8.2.4. Analysis of condition of optimal parameter selection . . . . . . . . 188

8.2.5. Comparison of the proposed way to select method parameters with known analogues . . 189

8.2.6. Estimate of integration method error of differential equations linear systems with constant coefficient matrix . . . . . . . 195

8.2.7. Algorithm of rigid linear systems numerical integration of differential equations with constant coefficient matrix . . . . . 200

8.2.8. Advantages of the algorithm . . . . . . 202

Part II. The random walk method. Solution of boundary-value problems

Chapter 9. Introduction to the random walk method (RWM) 207

Chapter 10. Numerical solution of the heat conductivity problems by means of the random walk method 209

10.1. Basic boundary-value problems of flat stationary heat conductivity theory . . . . . . 209

10.2. Main ideas of RWM for solution of the heat conductivity problems . . . . . . 211

10.3. The process of random walking over circles and its properties . . . . . . . . . . . 214 10.4. RWM algorithms for solution of the Dirichlet problem 219 
10.4.1. RWM algorithms on circles with displaced centers . . . . . . . . . 221

10.4.2. Traditional RWM algorithm . . . . . . . 230

10.4.3. RWM algorithms with partial integration ... 231

10.5. RWM algorithms for solution of the Neumann problem . . . . . . . . . . 242

10.5.1. Integral representations of the solution . . . . 242

10.5.2. RWM algorithm with displaced centers . . . . 245

10.6. Numerical results . . . . . . . . . . . . . . . 250

Chapter 11.The Monte-Carlo method applied to problems of plate curving 253

11.1. Statement of the problem . . . . . . . . . 253

11.2. Integral representations . . . . . . . . . . 256

11.3. Curving of supported plates with

linear piece-wise boundary . . . . . . . . . 263

11.3.1. Basic relations . . . . . . . . . . . 263

11.3.2. Traditional RWM method . . . . . . . . 266

11.3.3. RWM algorithm with displaced centers . . . . 268

11.3.4. RWM algorithm with partial integration ... 273

11.4. Curving of supported plates with arbitrary contour . . 277

11.4.1. Designing traditional algorithm in a formal way . . . . . . . . . . . 277

11.4.2. Designing of converging algorithm (modulation method) . . . . . . . . 279

11.4.3. Convergence conditions . . . . . . . . 285

11.4.4. Justification of the results obtained . . . . . 287

11.5. Curving of the fastened plates . . . . . . . . 300

11.5.1. Basic relations . . . . . . . . . . 300

11.5.2. Algorithm with partial integration ..... . 301

11.5.3. Some ways to improve efficiency of the algorithm . . . . . . . . 309

11.6. Results of numerical simulation . . . . . . . . . . 312

Chapter 12.The Monte-Carlo method applied to the flat problems of elasticity theory 315

12.1. Statement of the problem . . . . . . . . . 315

12.2. Description of RWM algorithm with partial integration ............ . 316

12.3. Finding displacements by the RWM . . . . . . . 317

12.4. Solution of test problems . . . . . . . . . . 320 
xviii

Chapter 13.Application of Monte-Carlo method towards finding of tensions at dangerous points of cog-wheels

13.1. Selection of computation models and setting of boundary conditions . . . . . . . . . . . 322

13.1.1. A rigid-rimmed cog-wheel . . . . . . . 323

13.1.2. A texible-rimmed cog-wheel . . . . . . . 323

13.2. Results of numerical simulation . . . . . . . . . 324

13.2.1. Rigid-rimmed cog-wheels . . . . . . . . . . . 324

13.2.2. Flexible-rimmed cog-wheels . . . . . . . 327

13.2.3. Problem of finding the best gear-cutting tool . 328

13.3. Some conclusions . . . . . . . . . . . . 330

Chapter 14. The spatial problem of heat conductivity 331

14.1. Defining relations . . . . . . . . . . . . . 332

14.2. Solution of the statistical problem of heat conductivity with boundary conditions of the second kind for a parallelepiped and a cube . . . . . . . 333

14.3. Construction of statistical estimates . . . . . . 336

14.4. Analysis of the algorithm convergence . . . . . . . 339

14.5. Results of numerical simulation . . . . . . . . . . 342

14.6. Statement of the final algorithm . . . . . . . . 344

Part III. Optimization of an FEM grid

Chapter 15.Introduction

Chapter 16.Optimal distribution of nodes for the problem of tension of a balk of variable section $\quad \mathbf{3 5 2}$

16.1. Statement of the problem . . . . . . . . . . 352

16.2. Solution of the problem by means of FEM . . . . . 353

Chapter 17.Optimization in general case 362

17.1. Asymptotically optimal density . . . . . . . . 362

17.2. Examples . . . . . . . . . . . . . 366

$\begin{array}{ll}\text { Chapter 18.Numerical simulation } & 368\end{array}$

18.1. Statement of the problem . . . . . . . . 368

18.2. Step approximation of distribution function .... . 368 
Chapter 19.BEMM optimal nodes with variable section, linear with respect to the length 373

19.1. Determinate case . . . . . . . . . . . . . 374

19.2. Optimal asymptotic density . . . . . . . . . 378

19.3. Construction of an optimal density of nodes distribution . . . . . . . . . . 380

Chapter 20. Connection between optimal determinate nodes and optimal density

Chapter 21.Results of numerical experiments 385

21.1. Simulation of determinate FEM solutions . . . . . 385

21.2. Simulation of stochastic FEM solutions . . . . . 388

$\begin{array}{ll}\text { Afterword } & 395\end{array}$

$\begin{array}{ll}\text { Bibliography } & 397\end{array}$

Index $\quad 413$ 